\title{
Pulmonary embolism secondary to uterine fibroid: A case report of a rare presentation
}

\author{
Ramy Ibrahim², Irina Dashkova², Myia Williams ${ }^{3 *}$, Trisha Khanna ${ }^{4}$, Andrzej Kozikowski ${ }^{3}$, Anna Dashkova², Renee Pekmezaris ${ }^{3}$
}

\begin{abstract}
Ibrahim R, Dashkova I, Williams M, et al. Pulmonary embolism secondary to uterine fibroid: A case report of a rare presentation. J Phlebol Lymphol. 2018;11(1):13-15.

Large Pulmonary Embolism with right heart strain in the presence of massive vaginal bleed is an uncommon presentation in middle aged women. Pulmonary embolism and Deep Vein Thrombosis are both rare and life-threatening conditions for women with uterine fibroids. We report a case of a 41-year-old Indian American woman with a history of uterine leiomyoma and menorrhagia, who presented to the emergency department with shortness of breath and 6 out of 10 retrosternal chest
\end{abstract}

pain. CT angiography showed massive bilateral pulmonary embolism with $5 \mathrm{~cm}$ benign right ovarian tumor and venous Doppler showed left deep vein thrombosis at femoral level. We successfully treated the patient with rapid intervention through thrombolysis followed by anticoagulation after initial stoppage of the bleeder points by targeted uterine artery embolization. Further studies can aim at early detection to minimize morbidity and mortality in females with uterine fibroids presenting with thromboembolic phenomenon.

Key Words: Uterine fibroids; Menorrhagia; Pulmonary embolism; Deep venous thrombosis; Ovarian benign tumor
Pulmonary embolism (PE) is a very common disorder and often an important cause of mortality and morbidity [1]. In the United States, $\mathrm{PE}$ is estimated to occur in approximately 600,000 patients annually and either cause or contribute to at least 50,000 to 200,000 deaths [1]. Many of the nonspecific clinical features of PE produce difficult diagnostic challenges for medicine, hence making the true incidence of PE unknown $[1,2]$. Additionally, PE occurs in approximately $50 \%$ of patients who have proximal DVT [3,4], and at least $80 \%$ of those diagnosed with PE also have DVT [4].

An extensive review of published data in the literature shows no consist differences in the occurrence of PE and DVT in men and women, despite evidence that the use of oral contraceptives and post-menopausal hormonal replacement have been associated with PE and DVT [5]. Other predisposing factors of DVT and resulting PE which are either acquired or inherited include, but are not limited too; pregnancy and post postpartum period, reduced mobility, deficiencies of blood coagulation agents, trauma, cancer and major surgery [4,5]. Tapson [3] also indicated advancing in age as a clear risk factor to PE and DVT, with a greater risk identified after the age of 40. In the same vein, Shiota et al., [6] also provided evidence which indicated that in women with uterine fibroids, larger tumors are more often than not complicated by DVT.

Uterine fibroids are the most widely occurring tumor in women of child bearing and reproductive age, yet they rarely cause acute complications [7]. A large fibroid can compress the pelvic venous system and surrounding structures which can lead to stasis and then DVT [7-10]. If uterine fibroids cause thromo-emobolism, it is expected that such an association is more commonly observed [7]. However, the occurrence of PE secondary to DVT and uterine fibroids is rarely reported in the literature, indicating that occurrences of such a phenomenon either goes unrecognized or unreported $[4,7]$.

We present a rare case of PE secondary to DVT and uterine fibroids which was successfully treated. In our case hyper functioning ovarian cyst led to increased incidence and size of uterine fibroids which led to secondary hypercoagulable state leading to deep vein thrombosis/pulmonary embolism. We address several related variables in such a rare but critical case so that further studies can aim at early detection to minimize morbidity and mortality in females with uterine fibroids presenting with thromboembolic phenomenon.

\section{CASE PRESENTATION}

A 41 year old G2 P2 immigrant woman of Indian heritage presented to our ED with heavy vaginal bleeding, moderate to severe anemia, shortness of breath, extreme weakness and dizziness with 6 out of 10 retrosternal chest pain since 2 days ago. The patient had a history of two caesarian sections and uterine leomyomas which occurred 3 years ago and caused recurrent episodes of menorrhagia which required 2 packed red blood cell transfusions. At the time the she was given iron tablets $325 \mathrm{mg}$, orally twice a day to control her anemia. There were no other significant past medical or family history reported.

The initial presentation in ER was highly suggestive of hemodynamic instability with persistently low systolic blood pressure range 70-80, as well as profound sinus tachycardia with EKG changes, and blood indices suggestive of moderate to severe anemia. On physical examination she was pale and extremely weak in moderate to severe distress with a blood pressure $80 / 50$, heart rate of $113-130 \mathrm{bpm}$, respiratory rate of $20-22$ breaths/min, a temperature of $98^{\circ} \mathrm{F}$, oxygen saturation $88-92 \%$, and body mass index 27.82. There were no icterus, petechiae, ecchymosis, or purpuric lesions, lymphadenopathy noted and lungs were clear to auscultation.

First we did a computed tomography angiogram (CT angiogram) on the patient. Cardiac examination revealed cardiac grade $3 / 6$ systolic flow murmur. The liver span was $8 \mathrm{~cm}$ and spleen was not palpable. Pelvic examination showed gush of vaginal bleeding and uterus was palpated at level $3 \mathrm{~cm}$ above umbilicus with prolapsed uterus and cervix dilated $4-5 \mathrm{~cm}$ $100 \%$ effacement. Was successfully reduced immediately by analgesia, and done by the gynecology in ER. No bleeding from other orifices was observed. Other findings include, a urine analysis positive for WBCs and leukocyte esterase, TSH 3.14, EKG showed sinus tachycardia with inverted T-waves in lead 2,V3,V4,V5,V6, immediate echo showed right heart strain with RV/ Lv ratio 1.93 .

Immediate CT angiography showed massive bilateral pulmonary embolism as well as $5 \mathrm{~cm}$ benign right ovarian tumor and later venous Doppler showed left DVT at femoral level. The uterine fibroid was $20 \times 23 \mathrm{~cm}$ sub-mucosal fibroid, which is nearly equivalent to a 20 week sized pregnant uterus. No biopsy was done on the ovarian tumor as the patient refused performing a biopsy for insurance and financial reasons. Further initial laboratory data is summarized in Table 1.

${ }^{1}$ Medical Director of Premier Medical Associates Group Orlando, Florida, United States, 2 Division of Geriatrics and Palliative Medicine, Northwell Health, 330 Community Drive, Manhasset, NY, 11030, United States, 3Department of Medicine, Northwell Health, 175 Community Drive, Manhasset NY, 11030, United States, 4Columbia Medical Center, 50 Haven Ave, New York, NY 10032, United States

Correspondence: Myia Williams, Department of Medicine, Northwell Health, 175 Community Drive, Manhasset NY, 11030, United States, Tel: (516)-600-1485; Fax: (516)-600-1508; E-mail: mwilliam26@northwell.edu ; Doi: 10.14303/1983-8905.1000051

Received: February 9, 2018, Accepted: February 27, 2018, Published: March 5, 2018 
Table 1

Summary of Initial Laboratory Results

\begin{tabular}{|c|c|}
\hline Parameter & Value \\
\hline Albumin & 3.4 \\
\hline Alkaline Phosphatase & 60 \\
\hline Amylase & 32 \\
\hline BUN ( Blood Urea Nitrogen) & 15 \\
\hline Calcium & 9.2 \\
\hline Carbon dioxide & 21 \\
\hline Chloride & 104 \\
\hline Creatinine & 0.77 \\
\hline CRP (C-reactive protein) & 0.83 \\
\hline DAU8 & negative \\
\hline D-dimers & 3.88 \\
\hline Globulin & 3.9 \\
\hline Glucose 114 & 114 \\
\hline Hemoglobin level & $7.9 \mathrm{~g} / \mathrm{dL}$ \\
\hline INR (International Normalized Ratio) & 1.3 \\
\hline Lipase 6 & 6 \\
\hline Mean corpuscular volume (MCV) & 62.4 femtolitre \\
\hline Platelet count & $203,000 / \mathrm{mm}^{3}$ \\
\hline Potassium, & 3.7 \\
\hline SGOT (Serum Glutamic-oxaloacetic Transaminase) & 14 \\
\hline SGPT (Serum glutamic Pyruvic Transaminase) & 33 \\
\hline Sodium & 137 \\
\hline Troponin & 0.12 \\
\hline TSH (Thyroid-Stimulating Hormone) & 3.14 \\
\hline
\end{tabular}

In our case, the patient was first stabilized with generous intravenous therapy (IV) levophed presser support, normal saline (NS) at $200 \mathrm{cc} / \mathrm{hr}$ and packed red blood cell (RBC) transfusion. She was admitted to the intensive care unit (ICU) under critical care medicine. The patient was given the option to under surgical embolectomy, but refused this option. Alternatively, the patient underwent immediate interventional radiology embolization of bilateral uterine arteries with 3 syringes 300-500 micron beads and 500-700 micron beads particles. Two syringes were used to stop the uterine bleed as well as guard against the anticipated massive bleed from thrombolysis. This was done at the same session by catheter directed thrombolysis of massive embolism by $24 \mathrm{mg}$ total tissue plasminogen activator (tPA) through pulmonary artery. Patient received 2 units of blood during the procedure and bleeding completely stopped few hours after. CBC was done every 4 hours with transfusion threshold if hemoglobin drops below 7 which never required further transfusion during her hospital stay. Next day infusion catheters were discontinued at bed side and patient had an IVC filter placed, was on heparin and was hemodynamically stable.

She was transferred in stable condition to a tertiary center for further care and possible elective hysterectomy as indicated by a gynecologist. Cardiology followed through with serial troponin, and marked clinical and troponin levels improvement after thrombolysis. In 1 day patient was able to maintain oxygen saturation $94-96 \%$ room air most of times as well as hemodynamic stability without any pressors or fluid support, with impressive overall improvement and blood systolic blood pressure (sbp) range 110-130, diastolic 60-80 during the rest of her stay. A repeat echo 4 days later was within normal limits and no evidence of remaining heart strain and EKG on discharge showed normal sinus rhythm rate 72 without T-wave abnormalities. Due to immediate initiation of thrombolytics hematology advised for outpatient hypercoagulable workup and patient risk stratification to prevent further relapse and to assist in defining new modalities in managing such rare but hard cases.

\section{DISCUSSION}

We presented a case of Hyper functioning ovarian cyst leads to increased incidence and size of uterine fibroids which leads to secondary hypercoagulable state leading to deep vein thrombosis/pulmonary embolism. Profound PE with marked acute chronic iron deficiency anemia secondary to right heart strain can be successfully managed if rapid intervention through thrombolysis followed by anticoagulation after initial stoppage of the bleeder points by targeted uterine artery embolization. Implementation of appropriate treatments such as thrombolysis and catheter-based therapy can reduce the risk of mortality, however other factors such as patient wishes, age, quality of life and co-morbidities should also be considered $[3,4]$. It is estimated that for at least $20-30 \%$ of women aged 30 years and over, uterine fibroids are the most common pelvic neoplasms [4,7]. Fifty percent of the women who experience uterine fibroids remain asymptomatic. On the other hand, the remaining $50 \%$ exhibit a variety of symptoms such as abnormal (usually heavy) bleeding, pelvic pressure or pain and urinary symptoms. Additionally, as the uterine fibroids increase in size there might be a significant increase in the occurrence of thrombo-embolism. As such there is necessary to preventive anticoagulation for larger uterine fibroid patients $[4,6,7]$. Other acute complications include torsion of subserosal pedunculated leiomyoma, hemorrhage, and urinary retention [4]. It is important to note that although acute complications of fibroids are rather rare, when they do occur and go undetected or untreated, acute complications can lead to the death of the woman [7].

Tapson [3] advised that being cognizant of the signs and symptoms of VTE significantly reduces diagnostic delays. Usually, patients with PE may have chest pain or dyspnea which may develop either suddenly in onset or evolve within days or weeks. Additionally, the patient may also show symptoms of cough, palpitations, and light-headedness which all may result from PE or from a concomitant illness [3]. In our case, the patient exhibited the abovementioned signs and symptoms. When PE or VTE is suspected, Tapson [3] suggested further testing must be considered. The extent of symptoms presented would depend on thrombo-emblosic burden. Large thrombi in one's periphery may evolve silently, present as symptomatic and may be fatal pulmonary embolism; on the contrary, a smaller emboli may be related to major symptoms, especially if cardiovascular reserve is poor [3].

Consequently, once PE is suspected, a careful assessment should be made based on the patient's history, physical examination, and known risk factors; likewise, well established standard studies such as electrocardiography, electrocardiogram (ECG) and chest radiograph should also be taken into consideration $[3,7]$. In situations where diagnosis can be difficult but PE is suspected, spiral CT can also be used for diagnosis [7]. To help identify the location of the thrombus, Doppler flow ultrasonography or venogram of lower extremities can be used. Similarly, magnetic resonance imaging (MRI) or computed tomography (CT) scan of the pelvis will assist to help define the mass of the fibroid as well as the location of the thrombus [7]. D-dimers are usually elevated in non-pregnant women; therefore, they will be useful [7]. In our case we followed similar procedures for diagnosis.

For women presenting with PE/VTE secondary to uterine fibroids, management can be challenging. The main goal for treatment is to deal with the VTE first, and then remove the fibroid [7]. There are few notable case reports in the literature of PE/DVT developing as a result of large uterine fibroids $[4,6,8,9,11,12]$. In the case studies reported, anti-coagulate associated with hysterectomy was the most commonly used treatment when DVT and PE developed secondary to uterine fibroids was diagnosed [4].

In the present case, we employed Gupta et al., [7] guiding principles in the management of a woman who presents with VTE as a result of large uterine fibroids. We took a multidisciplinary approach which involved senior practitioners in areas such as gynecology and hematology [7]. The hematologist was responsible for advising and guiding us on the issues of anticoagulation as a treatment option. Patients with PE who receive adequate anticoagulant therapy more often than not, it has been reported that the 3 -month overall mortality rate is $15-18 \%$. Since fertility was not a concern a hysterectomy was the surgical procedure utilized. Consequently, the patient was given post-operative care in the intensive care unit [7]. Following treatment of massive $\mathrm{PE}$ and anemia, symptoms rapidly resolved and patient remained stable during her hospital stay.

In our case we used herapin, as it is the recommended drug to start anticoagulation on patients with acute pulmonary thromboembolism (PTE). Using this drug proved to be effective in our patient as she was hemodynamically stable after drug was administered. There were no recognized risk factors such as herparin resistance (which is often encountered in patients who are acutely ill, patients with malignancies, and during peri- or post-partum period) [13,14] present in our patient.

The indication of pelvic compression by the enlarged uterus was an indication that a hysterectomy should be performed. Previous studies have reported that thrombolytic therapies as well as inferior vena cava filters are necessary treatments when PTE exists. Additionally, the management of such cases might prove to be even more difficult than those without preoperative DVT. As was done in our case, we used catheter directed thrombolysis which quickly reestablished pulmonary blood flow in the patient. The use of catheter fragmentation followed by intrapulmonary thrombolytic infusion is an aggressive technique which has often been used to achieve a rapid thrombus resolution, improving hemodynamic conditions in most patients [13]. 
Pulmonary embolism commonly originates from DVT which more often than not rapidly results in death even when appropriate treatment can reduce the risk of mortality [4]. Although there has been increase guidelines on the use of optimal therapy, including thrombolysis and catheter based therapies, has remained unclear. Therefore, when treating patients, we should consider other factors such as patient wishes, age, quality of life, as well as comorbidities for implementing proper therapy [4]. In our case we performed techniques and management of treatment as previously outlined in previous literature. We took into consideration the unique case of the patient as well as facilities and expertise which we had readily available.

\section{CONCLUSION}

Immediate aggressive intervention is required for all cases of massive pulmonary embolism especially in the setting of comorbidities as massive uterine bleed and acute or chronic blood loss anemia with right ventricular strain. Future studies should focus on early management of symptomatic fibroids and considering the necessary tests including D-dimers and/or venous Doppler to screen for thrombosis. In cases of worrisome leimyomas to lower both morbidity and mortality, more aggressive and prompt intervention is needed to deal with such critical cases, which are often diagnosed postmortem.

\section{DECLARATIONS}

Competing interests: The author(s) declare(s) that there is no conflict of interest regarding the publication of this article.

Ethical guidelines: All ethical guidelines were adhered to.

Consent for publication

The participant gave consent for publication.

Funding: None.

\section{REFERENCES}

1. Arcasoy SM, Kreit JW. Thrombolytic therapy of pulmonary embolism: a comprehensive review of current evidence. Chest. 1999;115:1695-707.
2. Uhland H, Goldberg LM. Pulmonary embolism: a commonly missed clinical entity. Diseases Chest. 1964;45:533-6.

3. Tapson VF. Acute pulmonary embolism. N Engl J Med. 2008;358:1037-52.

4. Khademvatani K, Rezaei Y, Kerachian A, et al. Acute pulmonary embolism caused by enlarged uterine leiomyoma: a rare presentation. Am J Case Rep. 2014;15:300-3.

5. White RH. The epidemiology of venous thromboembolism. Circulation. 2003; 107:I-4

6. Shiota M, Kotani Y, Umemoto M, et al. Deep-vein thrombosis is associated with large uterine fibroids. Tohoku J Exp Med. 2011;224:87-9.

7. Gupta S, Manyonda IT. Acute complications of fibroids. Best Pract Res Clin Obstet Gynaecol 2009;23:609-17.

8. Chong YS, Fong YF, Ng SC. Deep vein thrombosis in patients with large uterine myomata. Obstet Gynecol 1998;92:707.

9. Dekel A, Rabinerson D, Dicker D, et al. Thrombosis of the pelvic veins associated with a large myomatous uterus. Obstet Gynecol 1998;92:646-7.

10. Phupong V, Tresukosol D, Taneepanichskul S, et al. Unilateral deep vein thrombosis associated with a large myoma uteri. A case report. J Reprod Med. 2001;46:618-20.

11. Asciutto G, Mumme A, Marpe B, et al. Deep venous thrombosis in a patient with large uterine myomata. Case report. Minerva Ginecol 2008;60:451-3.

12. Tanaka H, Umekawa T, Kikukawa T, et al. Venous thromboembolic diseases associated with uterine myomas diagnosed before hysterectomy: a report of two cases. J Obstet Gynaecol Res. 2002;28:300-3.

13. Falcone M, Serra P. Massive pulmonary embolism in a woman with leiomyomatous uterus causing pelvic deep venous thrombosis. Ann Ital Med Int. 2005;20:104-7.

14. Grassi CJ, Swan TL, Cardella JF, et al. Quality improvement guidelines for percutaneous permanent inferior vena cava filter placement for the prevention of pulmonary embolism. J Vasc Interv Radiol. 2003;14:S271-5. 